\title{
ВОЗМОЖНОСТИ ПРИМЕНЕНИЯ ТЕРИПАРАТИДА В РЕАЛЬНОЙ КЛИНИЧЕСКОЙ ПРАКТИКЕ
}

\author{
Красулина К.А., Крюкова И.В., Древаль А.В., Полякова Е.Ю., Барсуков И.А.
}

ГБУЗ МО «Московский областной научно-исследовательский клинический институт им. М.Ф. Владимирского», Москва

ЦЕЛЬ: оценить эффективность терипаратида у больных с различными формами остеопороза в реальной клинической практике в Московской области.

МАТЕРИАЛЫ И МЕТОДЫ: В наблюдение было включено 83 пациента с остеопорозом: первичным (42 женщины в постменопаузе, 7 мужчин старше 50 лет), вторичным - связанным с приемом системных глюкокортикостероидов ( 25 женщин и 4 мужчины) и иного генеза ( 2 женщины, 3 мужчин). Средний возраст - 65 13 (22-92) лет. Диагноз остеопороза устанавливался по результатам денситометрии (DXA, T-критерий менее -2,5SD в позвоночнике и/или бедре) и/или на основании низкоэнергетического перелома бедра, тел позвонков или множественных переломов в анамнезе. Всем пациентам было назначено лечение терипаратидом 20 мкг в сутки.

Эффективность лечения оценивали по результатам DXA через 12 и 24 месяца и на основании наличия/ отсутствия новых случаев переломов. 8 пациентам с первичным остеопорозом исходно и через 3 месяца исследовался N-концевой пропептид проколлагена 1 типа (P1NP, норма для женщин в постменопаузе 20-115 нг/мл, для мужчин старше 25 лет - 20-85 нг/мл).

PЕзУЛЬтАТЫ: до начала лечения у 27 (32,53\%) пациентов были зарегистрированы множественные переломы, у 32 (38,55\%) - переломы тел позвонков, у 3 (3,61\%) - перелом бедра. У женщин с постменопаузальным остеопорозом количество переломов тел позвонков составило 20 (47,6\%) случаев, бедра 1 (2,4\%), множественные - 14 (33,3\%). Пациенты с остеопорозом, связанным с приемом глюкокортикостероидов, в 34,5\% ( $n=10)$ имели множественные переломы, в 34,5\% ( $n=10)$ - переломы тел позвонков, в 3,4\% (n=1) - бедра. Среди 7 мужчин с сенильным остеопорозом был 1 человек с множественными переломами (20\%), 2 - с переломами тел позвонков (40,0\%), 1 - с переломом бедра (20\%).

В группе женщин с постменопаузальным остеопорозом ( $\mathrm{n}=7)$ отмечено статистически значимое повы-

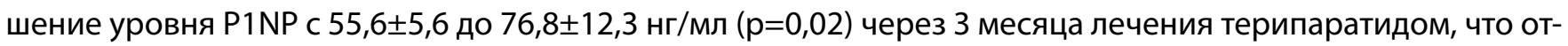
ражает раннюю эффективность терапии. У одного мужчины с сенильным остеопорозом так же выявлено увеличение маркера костеобразования с 49,3 до 61,8 нг/мл.

Через 2 года отмечена статистически значимая динамика минеральной плотности костной ткани (МПК) позвоночника у всех пациентов: Т-критерий и BMD соответственно повысились с исходных $-2,77 \pm 1,17$ SD и $0,77 \pm 0,14 г / \mathrm{cm}^{2}$ до $-2,57 \pm 1,11$ и 0,81 $\pm 0,12$ ( $p=0,002$ ). В проксимальном отделе бедра выявлено повышение МПК, но статистически не значимое. У пациенток с постменопаузальным остеопорозом, не имеющих сахарного диабета 2 типа $(\mathrm{n}=32)$, зарегистрирован прирост по Т-критерию в позвоночнике $\mathrm{c}-2,83 \pm 1,06 \mathrm{SD}$ до $-2,58 \pm 1,24 \mathrm{SD}(\mathrm{p}=0,014)$, в бедре - $\mathrm{c}-2,16 \pm 0,77 \mathrm{SD}$ до $-1,88 \pm 0,84 \mathrm{SD}$ ( $\mathrm{p}=0,046)$. В группе остеопороза, связанного с приемом глюкокортикостероидов, выявлено увеличение Т-критерия в позвоночнике $(-2,18 \pm 0,87$

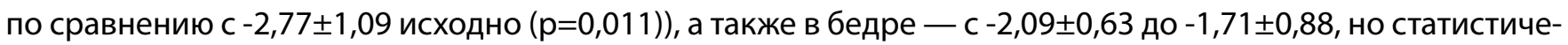
ски не значимое $(p=0,609)$. Не было зарегистрировано новых случаев переломов, а также нежелательных явлений.

ВЫВОдЫ: применение терипаратида в реальной клинической практике у пациентов как с первичным, так и вторичным остеопорозом в Московской области показало значимое повышение МПК без новых случаев патологических переломов на фоне лечения, а также характеризуется хорошим профилем переносимости. 